\title{
Pure erythroid leukemia: a reassessment of the entity using the 2008 World Health Organization classification
}

\author{
Wei Liu ${ }^{1}$, Robert $\mathrm{P}$ Hasserjian ${ }^{2}$, Ying $\mathrm{Hu}^{1}$, Liping Zhang ${ }^{3}$, Roberto $\mathrm{N} \mathrm{Miranda}^{1}$, \\ L Jeffrey Medeiros ${ }^{1}$ and Sa A Wang ${ }^{1}$ \\ ${ }^{1}$ Department of Hematopathology, The University of Texas MD Anderson Cancer Center, Houston, \\ TX, USA; ${ }^{2}$ Department of Pathology, Massachusetts General Hospital, Boston, MA, USA and \\ ${ }^{3}$ Department of Pathology, University of Massachusetts Medical Center, Worcester, MA, USA
}

\begin{abstract}
Pure erythroid leukemia (PEL) is rare, characterized by a neoplastic proliferation of erythroblasts. Given recent incorporation of molecular genetic findings and clinical features in the revised 2008 World Health Organization classification scheme of acute myeloid leukemia, we questioned if PEL still remains as a distinct subtype of acute myeloid leukemia. In this retrospective study, we identified 18 cases of acute leukemia with morphologic and immunophenotypic features of PEL. Following the current World Health Organization classification algorithm, these cases were classified as: 13 acute myeloid leukemia with myelodysplasia-related changes, 3 therapy-related acute myeloid leukemia, and 1 chronic myelogenous leukemia blast crisis, and one unclassifiable due to insufficient clinical information. All 16 cases with cytogenetic data harbored an extremely complex karyotype and the median overall survival of the 18 patients was 3 months (range, 1-7 months). This survival was significantly shorter than that of patients with acute erythroid leukemia, erythroid/myeloid subtype, or acute myeloid leukemia with myelodysplasia-related changes with erythroid predominance $(P<0.001)$. PEL is characterized as a neoplastic erythroid hyperproliferation with maturation arrest. E-cadherin emerged as the most sensitive and specific marker for immature erythroblasts, and was helpful in distinguishing PEL from other erythroid proliferations. Our study showed that the criteria for acute myeloid leukemia in the $\mathbf{2 0 0 8}$ World Health Organization system facilitate reclassification of PEL cases into other acute myeloid leukemia categories, mainly of acute myeloid leukemia with myelodysplasia-related changes. These new assigned categories fail to capture the distinct features of PEL, where the phenotype of PEL correlates with a very complex karyotype and an extremely aggressive clinical course.
\end{abstract}

Modern Pathology (2011) 24, 375-383; doi:10.1038/modpathol.2010.194; published online 19 November 2010

Keywords: acute erythroleukemia; cytogenetics; erythroid predominance; myelodysplastic syndromes; pure erythroid leukemia; survival

In 1917, Di Guglielmo first described a rare aggressive disease characterized by a neoplastic proliferation of immature cells committed solely to erythroid lineage with no increase in myeloblasts in bone marrow. This entity was designated as Di Gugliemo's disease, in contrast with Di Gugliemo's syndrome, the latter characterized by a stepwise progression of myelodysplastic syndromes (MDS) with erythroid predominance into erythroleukemia

Correspondence: Dr SA Wang, Department of Hematopathology, 1515 Holcombe Boulevard, Houston, TX 77030-4009, USA.

E-mail: swang5@mdanderson.org

Received 28 June 2010; revised 23 August 2010; accepted 23

August 2010; published online 19 November 2010 (erythroid/myeloid). ${ }^{1}$ Acute erythroleukemia (AEL) was not recognized as a subtype of AML in the initial French-American-British classification of AML (1976) but included in the 1985 revision of the $\mathrm{FAB}^{2}$ to define cases with erythroblasts comprising at least $50 \%$ of all bone marrow cells, and myeloblasts at least $30 \%$ of the non-erythroblasts (M6). Cases that fit the description of Di Guglielmo's disease were not officially recognized in either the initial or revised FAB classification schemes.

Despite being rare, others continued to study cases of Di Guglielmo's disease using a variety of terms including acute erythremic myelosis, erythroleukemia, M6 AML variant and M6b. ${ }^{3-6}$ In 1992, KowalVern et $a l^{6}$ proposed including erythroleukemias 
with $\geq 30 \%$ pronormoblasts, the most immature recognizable erythroid precursors, as a subtype of AML. They also proposed modifying the FAB system by renaming FAB-M6 as M6a, and cases that fit with Di Guglielmo disease as M6b. Pure erythroid leukemia (PEL) first appeared as a subtype of erythroleukemia in the 2001 WHO classification with the other being erythroid/myeloid. ${ }^{7}$ PEL remains as a category in the revised $2008 \mathrm{WHO}$ classification under AML not otherwise categorized (AML-NOS).

In the current WHO classification scheme, criteria for various types of AML have been modified and clinical presentation, cytogenetic and/or molecular genetic findings have been incorporated into AML classification. The new AML-with myelodysplasiarelated changes (AML-MRC) category has been introduced to include AML cases arising from previous MDS or myelodysplastic/myeloproliferative neoplasm (MDS/MPN); AML with MDS-related cytogenetic abnormalities; and AML with significant morphological dysplasia involving two and more lineages. AML cases with a history of cytotoxic therapy for an unrelated disease are designated as therapy-related AML (t-AML), while AML cases accompanied by recurring cytogenetic abnormalities are designated by their particular cytogenetic alteration regardless of the morphologic or immunophenotypic findings. Cases that do not fit into any of above well-defined categories are classified as AMLNOS, and further subcategorized by their morphologic and immunophenotypic characteristics. Thus, PEL cases are placed in the AML-NOS category only if they do not fit into any other specific categories such as t-AML, AML-MRC, or AML with recurrent cytogenetic abnormalities. ${ }^{8}$ We questioned if PEL would stay (or should stay) as a distinct subtype of AML under this new classification scheme.

Furthermore, PEL often posts great diagnostic challenges to pathologists, because left-shifted erythroid maturation can occur in various non-neoplastic or neoplastic conditions; and the leukemic erythroblasts lack clear lineage-defining markers and express myeloid-associated antigens such as CD117 and CD33. Various markers including glycophorins A, B, and C, hemoglobin, CD36, and spectrin have been used to mark immature erythroblasts in PEL; however, the results either lack consistency or specificity. ${ }^{5,9}$ E-cadherin is a cell adhesion molecule involved in the morphogenesis of multicellular organisms and maintenance of solid tissues. In the bone marrow, its expression appears to be limited to erythroid lineage, ${ }^{10}$ and mainly in immature erythroblasts. ${ }^{10-12}$ The diagnostic utility of E-cadherin in erythroid leukemia, however, has never been studied.

In this study, we identified cases that fulfilled the morphological and immunophenotypical criteria for PEL and reinvestigated these cases by applying the 2008 WHO classification criteria. In addition, we investigated the diagnostic utility of the adhesion marker, E-cadherin, in the workup of cases of PEL.

\section{Materials and methods}

\section{Study Group}

We searched the pathology archives at three medical centers (The University of Texas MD Anderson Cancer Center, Houston, TX, between 1995 and 2010; Massachusetts General Hospital, Boston, MA, between 2004 and 2008; University of Massachusetts Medical Center, Worcester, MA, between 1999 and 2007) for cases diagnosed as PEL defined as cases with: (1) $<5 \%$ myeloblasts and $\geq 80 \%$ erythroblasts in the bone marrow aspirate and where the erythroblasts were predominantly pronormoblasts and basophilic erythroblasts and $<10 \%$ late or mature erythroblasts; (2) the presence of sheets of immature erythroblasts on the bone marrow core biopsy or aspirate clot section; and (3) additionally, we included cases with $>50 \%$ erythroblasts, of which, $<10 \%$ were differentiating erythroblasts and most were undifferentiated/primitive blasts of confirmed erythroid lineage. ${ }^{5}$ Clinical information was obtained by review of the electronic medical records for history of any antecedent neoplasm, chemotherapy, radiation therapy, or cytopenia(s) as well as the treatment information and outcome. This study was approved by the institutional review board of all participating institutions. Overall survival was calculated from the day of diagnosis until death from any cause and was censored for patients known to be alive at the last follow-up.

The PEL cases were compared to a control group of 124 patients with AEL (also know as erythroid/ myeloid type) and 41 patients with AML-MRC, both of which had $\geq 50 \%$ erythroid bone marrow elements; these patients had been treated at the same institutions during a similar time period. The morphological, clinical, and cytogenetic features of these control cases have been published previously. ${ }^{13}$ The AEL cases with an overwhelming erythroid component $(>80 \%)(n=16)$ were compared to PEL for their bone marrow morphological and immunophenotypical features.

\section{Morphologic Assessment}

Bone marrow aspirate smears and aspirate clot and core biopsy specimens were assessed and the following pathologic parameters were scored: overall bone marrow cellularity was assessed based on the biopsy specimen; differential count (500 cells, or all available cells) on bone marrow aspirate smears; and percentages of dysplastic forms in the erythroid, myeloid, and megakaryocytic lineages on the bone marrow biopsy specimen and aspirate smears. The presence of dyspoiesis was based on the presence of $\geq 10 \%$ affected cells in a specific lineage, as suggested in the WHO classification.

Cytochemical stains including Prussian blue iron stain, periodic acid-Schiff reaction (PAS), myeloperoxidase (MPO), and $\alpha$-napthyl butyrate esterase were 
performed in all cases with available bone marrow aspirate smears. To quantify ring sideroblasts, 100 erythrocytes were counted manually. Peripheral blood smears from each case were also examined.

\section{Immunohistochemistry}

Immunohistochemical staining was performed on formalin-fixed, paraffin-embedded bone marrow core biopsy sections. After deparaffinization and rehydration in graded alcohols and xylene, endogenous peroxidase was blocked with hydrogen peroxide. Heat-induced epitope retrieval was performed by heating slides in EDTA buffer at $\mathrm{pH}$ 8.0, using a Black and Decker Handy Steamer Plus, and then cooled for $20 \mathrm{~min}$. The immunostaining was completed according to methods using the LSAB2 detection kit (DAKO), with appropriate positive and negative controls. Antibodies specific for CD33 (Novocastra, 1:200), CD34 (Biogenex, 1:100), CD117 (DAKO, Carpinteria, CA, 1:200), E-cadherin (DAKO, 1:100), Glut-1 (DAKO, 1:200), Glycophorin (DAKO, 1:200), MPO (Cell Marque, 1:2000) were used in the panel.

\section{Conventional Cytogenetics}

Conventional cytogenetic analysis was performed in three different laboratories using standard methods with minor differences in technique. In all cases, G-banded metaphase cells were prepared from unstimulated bone marrow aspirate cultures using standard techniques. Twenty metaphases were analyzed in all cases, if available, and the results reported using the International System for Human Cytogenetic Nomenclature. In some cases, a lesser number of metaphases were available, but in all cases the numbers of metaphases were adequate to issue a karyotype.

\section{Statistical Analysis}

Mann-Whitney test was used for numerical comparison between two groups. Fisher's exact test and $\chi^{2}$ test were applied for categorical variables. Patient survival was estimated by the Kaplan-Meier method from the date of bone marrow diagnosis until death from any cause or until the last patient follow-up. Survival curves were statistically compared by the log-rank test. Differences between two groups were considered statistically significant if $P$-values were $<0.05$ in a two-tailed test.

\section{Results}

\section{Patients}

A total of 18 patients met morphologic and immunophenotypic criteria for PEL, including 12 men and
6 women, with a median age of 66 years (range, 3393 years) (Table 1). This subset represents $13 \%$ of all erythroleukemia cases and $<1 \%$ of all AML cases diagnosed at the three institutions over the same time period. Patient 1 had a clinical history of chronic myelogenous leukemia (CML) treated with imatinib for 7 years before presenting with pancytopenia. Bone marrow biopsy revealed PEL, consistent with erythroid blast crisis of CML. Three patients had a history of malignancies treated with chemotherapy and/or radiation therapy: 1 diffuse large B-cell lymphoma, 1 plasma cell myeloma, and 1 glioma. Seven patients had a diagnosis of MDS for a median of 7 months (range, 1 month to 3 years) and presented with worsening cytopenias. The remaining six patients had no significant prior history and presented as de novo disease (Table 1). The control group consisted of 165 patients with AEL or AMLMRC patients (also see Materials and methods). The median age of these patients was 63 years (range, 12-90 years).

\section{Morphologic and Immunophenotypic Characteristics}

Peripheral blood smears were examined in all cases and showed circulating erythroblasts in four patients (22\%) (Figure 1a). Bone marrow aspirate smears were variable in quality due to hemodilution and the presence of smudge cells; overall erythroblasts represented $59-89 \%$ (median, 64\%) of total nucleated cells. The immature erythroblasts, pronormoblasts, and basophilic normoblasts, had round nuclei with fine chromatin, one to three nucleoli, and deeply basophilic, sometimes vacuolated cytoplasm. Occasional binucleated or multinucleated forms were present. These immature erythroid forms were $40-83 \%$ (median, $50 \%$ ) of all nucleated erythroid elements, or 25-81\% (median, $40 \%$ ) of all nucleated bone marrow cells. In two cases, the immature erythroid cells were small to medium in size, with a scant amount of deeply basophilic cytoplasm, morphologically resembling undifferentiated AML blasts (Figure 1b). In these two cases, the blasts comprised 39 and $50 \%$ of the total nucleated cells, and 59 and 64\% erythroblasts. Myeloblasts represented 1-5\% (median, 3\%) of all bone marrow cells. Dyserythropoiesis was present in 50\% cases, while dysgranulopoiesis and megakaryocytic dyspoiesis were present in 20 and $60 \%$ of the cases, respectively (Table 2). In all 16 cases of PEL assessed, both the immature and mature erythroblasts were negative for MPO and $\alpha$ naphtyl butyrate esterase, and PAS demonstrated intense cytoplasmic globules in all cases (100\%) (Figure 1c). Ring sideroblasts were present in 5 of 10 cases $(50 \%)$ stained, comprising $5-70 \%$ of all erythroid elements (in both immature and mature forms).

The bone marrow aspirate clot and core biopsy specimens in all cases were hypercellular. 


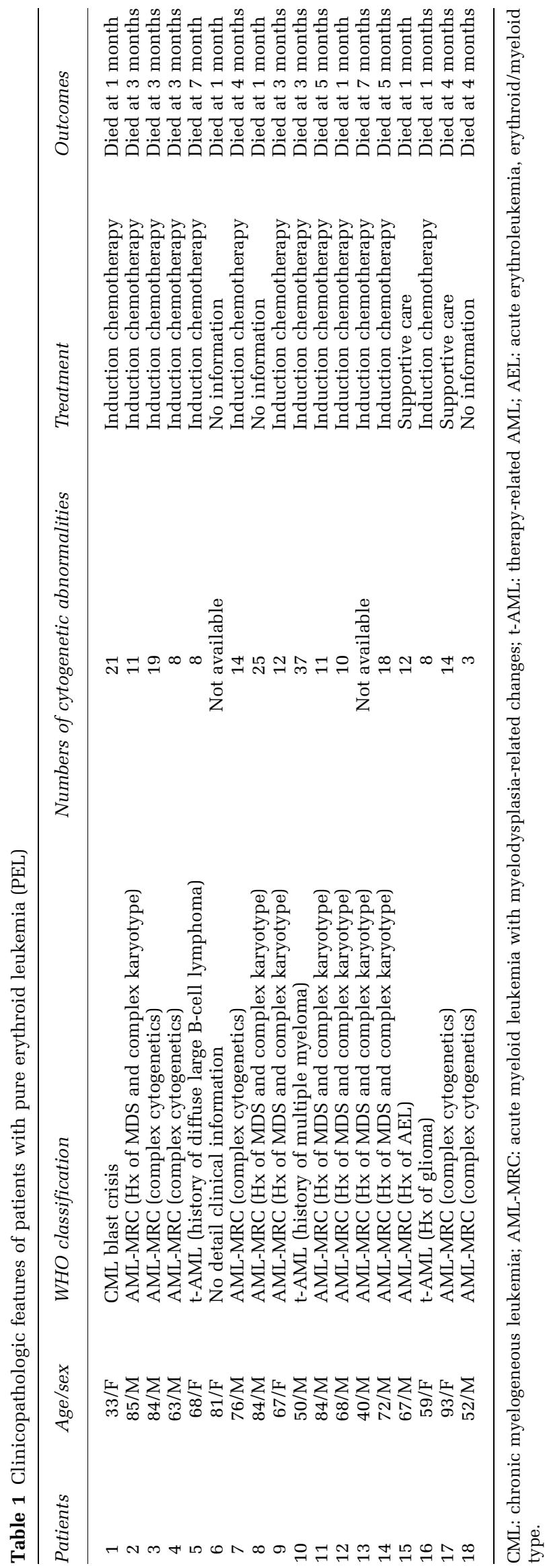

The median cellularity was $80 \%$ (range, 60-90\%) and showed erythroid predominance with sheets of immature cells (Figure 1d). The infiltrating immature erythroid cells were larger than typical AML blasts, and had somewhat more open or paler chromatin. Bone marrow fibrosis was present in only one case and resulted in a hypocellular aspirate. In general, bone marrow biopsy better and more reliably showed erythroid predominance and immaturity of these cells compared with bone marrow aspirate smears as the latter were often affected by hemodilution, smudge cells, and staining quality.

The control group of 16 AEL cases showed overwhelming erythroid hyperproliferation ( $\geq 80 \%$ erythroid elements) and maturation of the erythroid elements. The immature erythroid precursors, pronormoblasts and basophilic erythroblasts, averaged $8 \%$ (range, 3-25\%) and morphologically resembled normal pronormoblasts and basophilic erythroblasts. Ring sideroblasts were present in three cases $(19 \%)$, mostly present in late normoblasts. Dyserythropoiesis was invariably present $(100 \%)$, whereas dysgranulopoiesis and dysmegakaryoccytopoiesis were present in 50 and $81 \%$ cases, respectively. Coarse globular cytoplasmic stain by PAS was present in 4 out of 16 cases (25\%), mostly in immature cells, with some in mature erythroblasts, but in a smaller percentage of cells than PEL. Myeloblasts represented $4-18 \%$ of total cells and $25-70 \%$ of the non-erythroid cells (by definition, $\geq 20 \%$ ). Bone marrow biopsy demonstrated erythroid predominance with immature cells in small clusters.

Immunohistochemical analysis (Figure 2) including MPO, CD33, CD117, CD34, glycophorin A, Glut-1, and E-cadherin was performed on 14 PEL cases and 16 AEL with $\geq 80 \%$ erythroids (Table 2). Immature erythroblasts in PEL were negative for CD34 (Figures 2b and 3f) and MPO, but a subset of the cells was positive for CD33 and CD117 (Table 2). The most striking results were with the antiE-cadherin antibody. E-cadherin showed weak-tostrong membrane reactivity in sheets of large, immature erythroblasts in 13 of 14 (93\%) cases of PEL assessed, including two cases with undifferentiated morphology but much less number in AEL (Figures 2c and g). Glycophorin A and Glut-1 stained mainly the mature erythroblasts that were frequent in AEL and sparse in PEL (Figures 2d and h), but was weakly positive in the immature erythroblasts in three cases $(21 \%)$ of PEL. In contrast, the results in the AEL control were those expected as reported by others. Myeloblasts were positive for MPO, CD33, CD117, and CD34 (variably).

\section{Cytogenetic Data}

The results of conventional cytogenetic analysis were available for 16 PEL patients. The bone marrow 

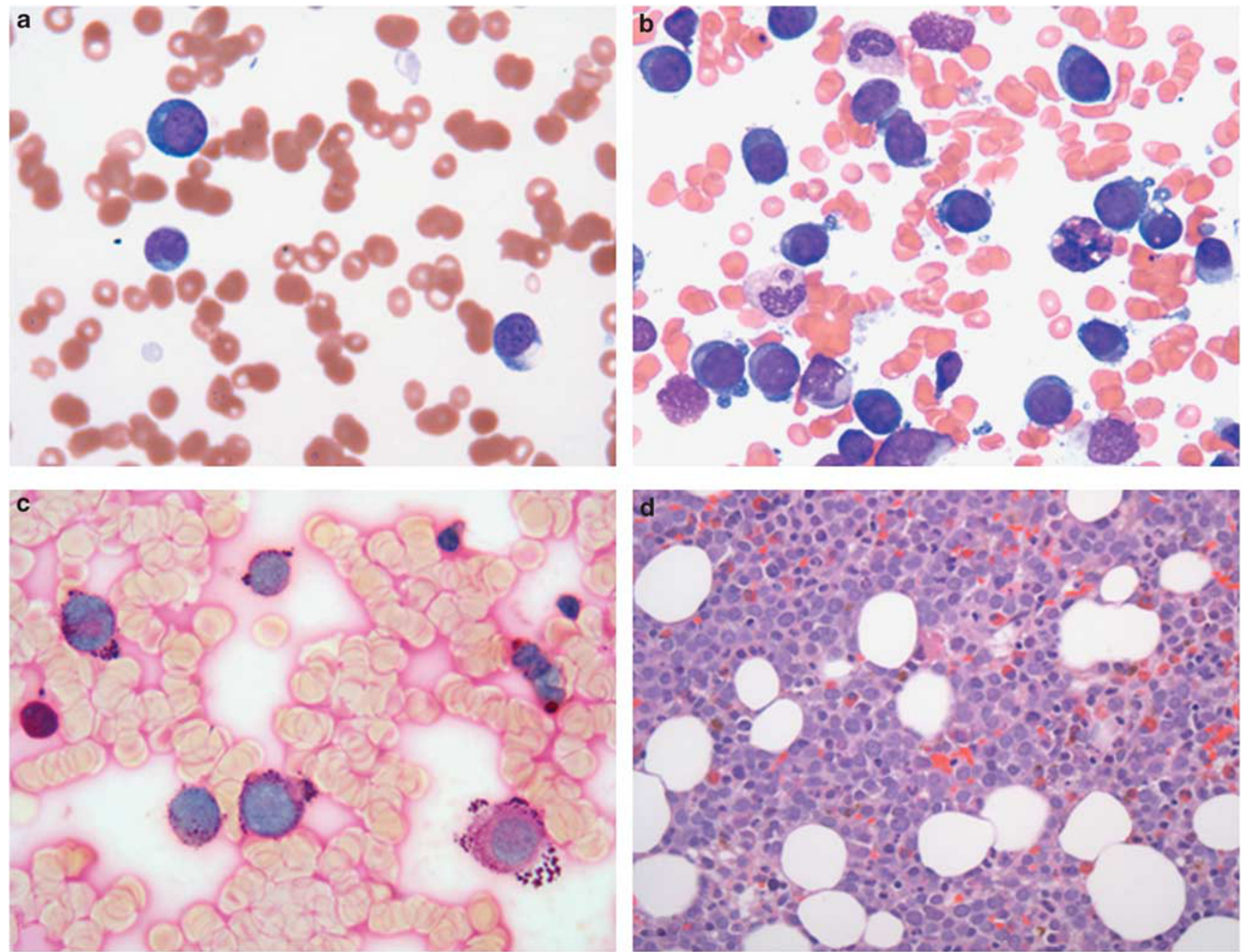

Figure 1 Morphologic features of pure erythroid leukemia. (a) Circulating erythroblasts; (b) primitive/undifferentiated erythroblasts on bone marrow aspirate smear; (c) cytoplasmic blocks/globules by periodic acid-Schiff reaction (PAS) stain; (d) bone marrow biopsy shows a monotonous leukemic infiltrate.

studied all showed an extremely complex karyotype, with a median of 12 abnormalities (range, 3-37). These abnormalities most frequently involved chromosomes $5(11 / 16 ; 69 \%), 7(9 / 16 ; 56 \%), 17$ $(7 / 16 ; 44 \%)$, and $19(6 / 16 ; 38 \%)$. All patients belonged to the unfavorable cytogenetic risk group according to the United Kingdom Medical Research Council criteria for AML (UKMRC). ${ }^{14}$

\section{Therapy and Clinical Outcomes}

The treatment information is listed in Table 1. The median overall survival of the 18 PEL patients was 3 months. All patients expired within a follow-up period of 7 months. We compared the survival of these 18 PEL patients with the control group consisting of 124 patients with AEL and 41 patients with AML-MRC with $\geq 50 \%$ erythroblasts. ${ }^{13}$ Cytogenetics information for our control group was available in 163 patients, of which, 58 (36\%) were assigned to the UKMRC unfavorable risk group, and the remaining 105 assigned to the intermediate risk group. The overall survival of PEL patients was significantly inferior to the entire control group (median overall survival, 3 months vs 12 months; $P<0.0001)$ as well as to the 58 control group patients with unfavorable cytogenetics (median overall survival, 3 months vs 6.5 months; $P<0.0001$ ) (Figure 3).

\section{Classification Using the 2008 WHO System}

Using the criteria of the 2008 WHO classification, 13 of the cases were reclassified as AML-MRC, 3 were t-AML, and 1 was CML erythroid blast crisis. The 13 AML-MRC cases were classified as such because of a history of MDS $(n=7)$ or the presence of specific cytogenetic findings $(n=6)$ (Table 1). One patient with PEL (case 6) could not be reassessed using the WHO system because of insufficient clinical information. Overall, although all the cases fulfilled the morphologic and immunophenotypic criteria of 
Table 2 Comparison of bone marrow features of pure erythroid leukemia (PEL) and acute erythroleukemia (erythroid/myeloid type) (AEL) with a prominent erythroid component $(\geq 80 \%)$

\begin{tabular}{|c|c|c|}
\hline & $\begin{array}{l}\text { AEL (with } \geq 80 \% \\
\text { erythroid elements) }\end{array}$ & $P E L$ \\
\hline Age (range, median) & $37-77,62$ years & 33-93, 68 years \\
\hline Gender (M/F) & $9 / 7$ & $12 / 6$ \\
\hline Bone marrow cellularity & $20-100 \%$, Median $80 \%$ & $60-90 \%$, Median $80 \%$ \\
\hline $\begin{array}{l}\text { Presence of ring sideroblasts }(n / n) \% \text { of ring } \\
\text { sideroblasts (range) }\end{array}$ & 3/16 Patients $5-80 \%$ & 5/10 Patients $5-70 \%$ \\
\hline Coarse cytoplasmic granules by PAS stain & 4/16 Patients & 14/14 Patients \\
\hline \multicolumn{3}{|l|}{ Dysplasia of hematopoietic lineages } \\
\hline Dyserythropoiesis & 16/16 Patients & 5/10 Patients \\
\hline Dysgranulopoiesis & 8/16 Patients & 2/10 Patients \\
\hline Dysmegakaryopoiesis & 13/16 Patients & 6/10 Patients \\
\hline Increased reticulin fibrosis & 1/16 Patients & 1/10 Patients \\
\hline \multicolumn{3}{|l|}{ Pattern of immunohistochemical stains } \\
\hline CD34 & $5-20 \%$ & $<5 \%$ \\
\hline Glycophorin & $\begin{array}{l}\text { Highlights majority of erythroid } \\
\text { elements }\end{array}$ & $\begin{array}{l}\text { Most staining a small subset of } \\
\text { mature erythrocytes, three } \\
\text { cases staining immature } \\
\text { erythroblasts }\end{array}$ \\
\hline E-cadherin & $\begin{array}{l}\text { Small subset erythroblasts } \\
\text { positive }\end{array}$ & $\begin{array}{l}\text { Majority of erythroblasts } \\
\text { positive }\end{array}$ \\
\hline Unfavorable cytogenetics & 14/16 Patients & 16/16 Patients \\
\hline
\end{tabular}

PEL, none of them would be retained in the PEL category according to the 2008 WHO Classification algorithm.

\section{Discussion}

In this study, we showed that PEL cases shared a number of distinctive features including hyperproliferation of immature erythroblasts with maturation arrest in the bone marrow, an extremely complex karyotype, and an aggressive clinical course with a truly short overall survival. However, using the 2008 WHO classification, where clinical preconditions and molecular cytogenetics 'trump' morphologic and immunophenotypic characteristics, all cases in our series were reassigned to other categories, mostly AML-MRC or t-AML, leaving PEL a nearly non-existent entity. Is this the best resolution for PEL cases? Or there is advantage in keeping the PEL category?

We started with the definition of PEL. PEL in the $2008 \mathrm{WHO}^{8}$ is defined as 'a neoplastic proliferation of immature cells committed exclusively to the erythroid lineage $(\geq 80 \%$ of bone marrow cells) with no evidence of a significant myeloblastic component.' However, in the study referred by the 2008 WHO for the definition of PEL consisting of eight cases of minimally differentiated erythroleukemia, the inclusion criteria were $\geq 50 \%$ immature erythroblasts with $<10 \%$ 'differentiating' forms. ${ }^{5}$ In contrary, Mazzella and colleagues ${ }^{6,15,16}$ have consistently used $30 \%$ pronormoblasts of erythroid precursors as the cutoff to define PEL. In 2002,
Domingo-Claros et $a l^{17}$ studied 62 cases of acute erythroid neoplastic proliferation by using the 2001 WHO criteria. Fifty-three cases were classified as AEL and two cases of PEL, leaving seven cases unclassifiable. Two of the seven unclassifiable cases had undifferentiated erythroblasts of 42 and $40 \%$, respectively. In our series, the immature cells in PEL were clearly neoplastic and demonstrated a leukemic infiltrative pattern on bone marrow core biopsy; however, the total erythroblasts were $\leq 80 \%$ in nine cases, while the immature erythroid components ranged from 40 to $88 \%$ of all erythroid forms. We acknowledge that the strict WHO definition for PEL helps avoid confusion with other conditions of erythroid hyperproliferation with a left-shifted maturation seen in both non-neoplastic and neoplastic conditions., ${ }^{4,18}$ However, if the immature erythroblasts represent a monotonous, overtly neoplastic proliferation, we question the rationale for the requirement of $\geq 80 \%$ erythroblasts to qualify a case for PEL as other types of AML (including megakaryocytic) require only $\geq 20 \%$ blasts.

This also brought up the second question 'is PEL a true leukemia'. PEL has been suggested to represent the extreme end in the spectrum of MDS with erythroid predominance and a poor karyotype because of the absence of increased myeloblasts. For this reason, PEL was left out in the initial and revised FAB-AML classifications. We showed in our cases that PEL is indeed of a 'true erythroleukemia' where proliferation and differentiation are both affected, akin to the double-hit models proposed to underlie other types of AML with recurrent genetic abnormalities. ${ }^{19-21}$ Conversely, while AEL is also a 

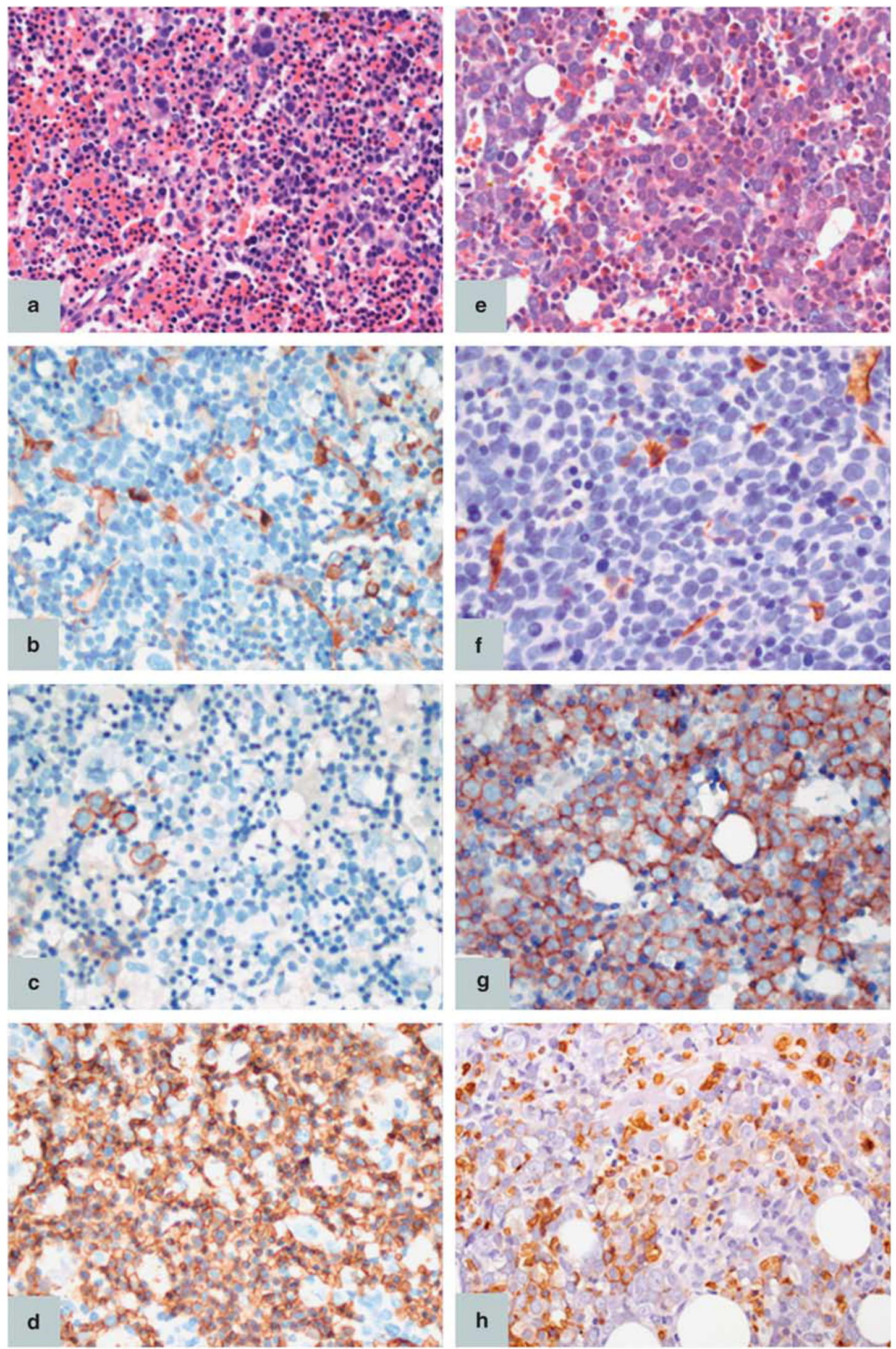

Figure 2 Morphological and immunophenotypical features of pure erythroid leukemia (PEL) (e-h) in comparing to acute erythroid leukemia (AEL) with a prominent erythroid component ( $\geq 80 \%$ erythroid) (a-d). (a, e) Bone marrow biopsy; (b, f) CD34; (c, g) E-cadherin; (d, h) glycophorin. 


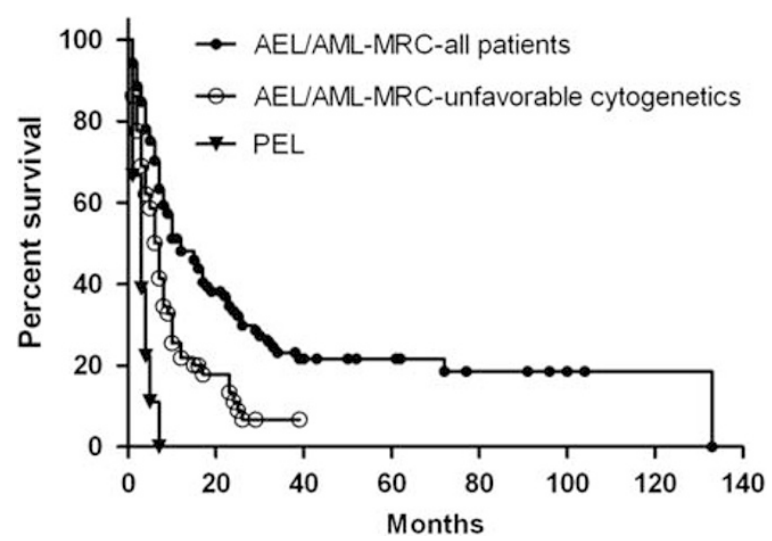

Figure 3 Overall survival of patients with pure erythroid leukemia (PEL). The median overall survival of patients with PEL was 3 months (1-7 months), significantly inferior to patients with acute erythoid leukemia (erythroid/myeloid) and acute myeloid leukemia with $\geq 50 \%$ erythroblasts, either as a cohort ( $n=165$, median 12 months, $P<0.0001$ ), or stratified by the unfavorable risk cytogenetic group by the United Kingdom Medical Research Council criteria for AML (UKMRC) $(n=58$, median 7 months, $P<0.0001)$.

clonal stem cell neoplasm, the erythroblasts in AEL are dysplastic but still capable of differentiating. In fact, we and others have shown that AEL and MDS with erythroid predominance are a continuum where karyotype rather than an arbitrary myeloblast cutoff represents the most important prognostic factor in this group of myeloid neoplasms. ${ }^{13,22,23}$ It is notable that the karyotypic abnormalities, despite all being classified in the unfavorable category, were more complex in PEL than AEL or MDS with erythroid predominance. We speculate that certain molecular cytogenetic alterations are required to promote a neoplastic erythroid hyperproliferation; perhaps yet more complex molecular cytogenetic alterations underlie the block in erythroid maturation that characterizes PEL.

We showed that PEL can be de novo, evolving from MDS or AEL, therapy-related or as CML blast crisis; however, regardless the initial clinical presentations, this phenotype of AML denotes a complex karyotype with frequent abnormalities of chromosomes 5 and 7 and a universally poor outcome in affected patients. These results are similar to our findings in AEL and MDS with erythroid predominance, in which a history of preexisting MDS or cytotoxic therapy are not independently related to outcomes. ${ }^{13}$ Similar findings have been reported in acute megakaryocytic leukemia in adults, which represents an aggressive variant AML regardless of the preconditions. ${ }^{24}$ We readily acknowledge that morphologic appearance is less ideal than well-defined molecular markers, like DNA, mRNA, and microRNA profiles to categorize diseases for many reasons, but those markers are not currently available.

In PEL, because primitive/undifferentiated erythroid often lack of lineage-specific markers, it often poses diagnostic challenges to pathologists. In this study, we showed that E-cadherin was specific for erythroid lineage in bone marrow, ${ }^{11}$ and its reactivity confined to the very immature erythroblasts and retained in PEL. Glycophorin A expresses mainly in terminal stages of erythroid differentiation, and only aberrantly expresses in immature erythroblasts in 3/14 PEL cases in our series. Other erythroid markers, such as glycophorin C, and CD36 have been shown to label relatively early stages of erythroblasts $^{9,25}$; however, they are not entirely specific to erythroid lineage. ${ }^{25,26}$ The markers $\mathrm{RhD}$ antigen and spectrin are expressed in immature erythroid cells but have not been investigated in PEL. ${ }^{5,27}$ Nevertheless, the markers chosen to assess erythroid leukemia should be able to label primitive or minimally differentiated erythroblasts, which could be mistaken as undifferentiated AML (FABM0) due to their expressions of CD117 and CD33 myeloid-related antigens. The combination of E-cadherin with glycophorin A can effectively separate immature vs mature erythroid precursors in bone marrow biopsy sections.

In summary, we suggest that the phenotype of PEL, although not well understood at the molecular level, conveys useful information that is not adequately captured in the current WHO classification. We showed that PEL represents a neoplastic erythroid hyperproliferation with maturation arrest, biologically more akin to a true erythroleukemia than AEL. The morphologic and immunophenotypic features of PEL define a form of acute leukemia with a highly complex karyotype and a very poor prognosis, which requires aggressive clinical management.

\section{Disclosure/conflict of interest}

The authors declare no conflict of interest.

\section{References}

1 Di Guglielmo G. Le Malattie Eritremiche ed Eritroleucemiche. Il Pensiero Scientifico: Rome, 1962.

2 Bennett JM, Catovsky D, Daniel MT, et al. Proposed revised criteria for the classification of acute myeloid leukemia. A report of the French-American-British Cooperative Group. Ann Intern Med 1985;103: 620-625.

3 Hasserjian RP, Howard J, Wood A, et al. Acute erythremic myelosis (true erythroleukaemia): a variant of AML FAB-M6. J Clin Pathol 2001;54:205-209.

4 Kowal-Vern A, Mazzella FM, Cotelingam JD, et al. Diagnosis and characterization of acute erythroleukemia subsets by determining the percentages of myeloblasts and proerythroblasts in 69 cases. Am J Hematol 2000;65:5-13.

5 Garand R, Duchayne E, Blanchard D, et al. Minimally differentiated erythroleukaemia (AML M6 'variant'): a rare subset of AML distinct from AML M6. Groupe 
Francais d'Hematologie Cellulaire. Br J Haematol 1995; 90:868-875.

6 Kowal-Vern A, Cotelingam J, Schumacher HR. The prognostic significance of proerythroblasts in acute erythroleukemia. Am J Clin Pathol 1992;98:34-40.

7 Harris NL, Jaffe ES, Diebold J, et al. World Health Organization classification of neoplastic diseases of the hematopoietic and lymphoid tissues: report of the Clinical Advisory Committee meeting-Airlie House, Virginia, November 1997. J Clin Oncol 1999;17: 3835-3849.

8 Arber DA, Orazi A, Porwit A, et al. WHO Classification of Tumours of Haematopoietic and Lymphoid Tissues. Lyon: IARC press, 2008.

9 Sadahira Y, Kanzaki A, Wada H, et al. Immunohistochemical identification of erythroid precursors in paraffin embedded bone marrow sections: spectrin is a superior marker to glycophorin. J Clin Pathol 1999; 52:919-921.

10 Armeanu S, Buhring HJ, Reuss-Borst $\mathrm{M}$, et al. E-cadherin is functionally involved in the maturation of the erythroid lineage. J Cell Biol 1995;131:243-249.

11 Armeanu S, CA MU, Klein G. Involvement of E-cadherin in the development of erythroid cells; subject heading. Hematology 2000;5:307-316.

12 Buhring HJ, Muller T, Herbst R, et al. The adhesion molecule E-cadherin and a surface antigen recognized by the antibody 9C4 are selectively expressed on erythroid cells of defined maturational stages. Leukemia 1996;10:106-116.

13 Hasserjian RP, Zuo Z, Garcia C, et al. Acute erythroid leukemia: a reassessment using criteria refined in the 2008 WHO classification. Blood 2010;115:1985-1992.

14 Grimwade D, Walker H, Oliver F, et al. The importance of diagnostic cytogenetics on outcome in AML: analysis of 1612 patients entered into the MRC AML 10 trial. The Medical Research Council Adult and Children's Leukaemia Working Parties. Blood 1998;92: 2322-2333.

15 Mazzella FM, Alvares C, Kowal-Vern A, et al. The acute erythroleukemias. Clin Lab Med 2000;20:119-137.

16 Mazzella FM, Kowal-Vern A, Shrit MA, et al. Acute erythroleukemia: evaluation of 48 cases with reference to classification, cell proliferation, cytogenetics, and prognosis. Am J Clin Pathol 1998;110:590-598.

17 Domingo-Claros A, Larriba I, Rozman M, et al. Acute erythroid neoplastic proliferations. A biological study based on 62 patients. Haematologica 2002;87:148-153.

18 Wang SA, Tang G, Fadare O, et al. Erythroid-predominant myelodysplastic syndromes: enumeration of blasts from nonerythroid rather than total marrow cells provides superior risk stratification. Mod Pathol 2008;21:1394-1402.

19 Goldberg SL, Noel P, Klumpp TR, et al. The erythroid leukemias: a comparative study of erythroleukemia (FAB M6) and Di Guglielmo disease. Am J Clin Oncol 1998;21:42-47.

20 Park S, Picard F, Dreyfus F. Erythroleukemia: a need for a new definition. Leukemia 2002;16:1399-1401.

21 Acquaviva C, Gelsi-Boyer V, Birnbaum D. Myelodysplastic syndromes: lost between two states? Leukemia 2010;24:1-5.

22 Olopade OI, Thangavelu M, Larson RA, et al. Clinical, morphologic, and cytogenetic characteristics of 26 patients with acute erythroblastic leukemia. Blood 1992;80:2873-2882.

23 Honda Y, Manabe A, Tsuchida M, et al. Clinicopathological characteristics of erythroblast-rich RAEB and AML M6a in children. Int J Hematol 2008;88:524-529.

24 Oki Y, Kantarjian HM, Zhou X, et al. Adult acute megakaryocytic leukemia: an analysis of 37 patients treated at M.D. Anderson Cancer Center. Blood 2006; 107:880-884.

25 Villeval JL, Le Van Kim C, Bettaieb A, et al. Early expression of glycophorin C during normal and leukemic human erythroid differentiation. Cancer Res 1989;49:2626-2632.

26 Solary E, Casasnovas RO, Campos L, et al. Surface markers in adult acute myeloblastic leukemia: correlation of CD19+, CD34+ and CD14+/DR-phenotypes with shorter survival. Groupe d'Etude Immunologique des Leucemies (GEIL). Leukemia 1992; 6:393-399.

27 Southcott MJ, Tanner MJ, Anstee DJ. The expression of human blood group antigens during erythropoiesis in a cell culture system. Blood 1999;93:4425-4435. 